\title{
Enhanced Recovery After Surgery (ERAS) para operación cesárea.
}

\author{
Full Title Enhanced Recovery After Surgery (ERAS) for Cesarean section. \\ https://doi.org/10.25237/carsach2020.14
}

Alejandro Jankelevich ${ }^{1}$ Hector J. Lacassie ${ }^{2}$

${ }^{1}$ Departamento de Anestesiología y Medicina Perioperatoria Escuela de Medicina, Facultad de Medicina Universidad de Chile

2 División de Anestesiología Escuela de Medicina, Facultad de Medicina Pontificia Universidad Católica de Chile

Autor corresponsal:

Hector J. Lacassie: lacassie@med.puc.cl

ORCID ID: https://orcid.org/0000-0001-5758-4113

Palabras Claves: Cesárea, Protocolo ERAS, Preoperatorio, Intraoperatorio, Postoperatorio

Key Words: Cesarean section. ERAS Protocol, Preoperative, Intraoperative, Postoperative

\section{Puntos clave}

- La prevalencia de cesáreas ha aumentado a nivel mundial durante las últimas décadas. Actualmente, Sudamérica es el continente con mayor tasa de cesáreas.

- Cualquier intervención que disminuya los costos de la cesárea, tendrá un gran impacto en la salud pública.

- Recientemente se publicaron las recomendaciones de la Sociedad ERAS para aplicar ERAS en cesárea.

- El protocolo ERAS tiene como objetivo estandarizar la atención perioperatoria, reduciendo la variabilidad en la atención, favoreciendo la creación de una vía de atención específica basada en la evidencia para el progreso de los resultados maternos y fetales, apuntando a la mejoría continua del proceso

- Realizamos un análisis crítico de las recomendaciones propuestas y vemos la aplicabilidad de estas guías en Chile.

- La implementación internacional del protocolo ERAS en cesárea, no presenta mayor readmisión al hospital.

\section{Resumen}

Hace más de diez años que la ERAS (Enhanced Recovery After Surgery) Society comenzó a publicar protocolos ERAS para diferentes cirugías. Recientemente, se publicó el protocolo ERAS para la operación cesárea. Para realizar sus recomendaciones, la ERAS Society reúne a un comité de expertos que realiza una extensa búsqueda de literatura y publica sus recomendaciones basadas en evidencia. En este artículo nosotros realizamos un análisis crítico de las recomendaciones y de la literatura que respalda estas recomendaciones. También, revisamos la experiencia inter- 
nacional de la implementación de ERAS en cesárea, además de realizar una encuesta a obstetras, anestesiólogos y neonatólogos sobre la actualidad nacional en la implementación de ERAS para cesárea en Chile.

\begin{abstract}
It has been more than ten years since the ERAS (Enhanced Recovery After Surgery) Society began to publish ERAS protocols for a variety of surgeries. Recently, they published the ERAS protocol for cesarean section. To generate their recommendations, the ERAS Society gathers a panel of experts that review the available literature, analyze it and publish their recommendations. In this article, we critically analyze the cesarean section ERAS protocol, recommendations and the literature they base their conclusions on. We examine the international experience in implementing this protocol and we also present the results of a survey performed on Chilean obstetricians, anesthesiologists and neonatologists about ERAS implementation in Chile.
\end{abstract}

\title{
Introducción
}

La operación cesárea es efectiva para prevenir la mortalidad materna y neonatal. La Organización Mundial de la Salud (OMS) recomienda que las tasas de cesárea no deben exceder de 15 por 100 nacidos vivos para optimizar los resultados maternos y neonatales, aunque recientes análisis han demostrado que tasas nacionales de cesárea de aproximadamente 19 por 100 nacidos vivos se asocian a una menor mortalidad materna y neonatal.(1) Varias publicaciones demuestran que tasas mayores no disminuyen la mortalidad, pero si aumentan los costos en salud.(2)

A pesar de las recomendaciones, la prevalencia de cesáreas ha aumentado a nivel mundial durante las últimas décadas. Actualmente, Sudamérica es el continente con mayor tasa de cesáreas $(42,9 \%)$, seguido por Norteamérica (32,3\%), Oceanía (31,1\%), Europa (25\%) y Asia (19,2\%).(3) En Chile, en 2014 la tasa de cesárea fue de 44,7\% con un total de 184.000 nacimientos, con 82.000 cesáreas aproximadamente.(4) En 2015, esta tasa aumentó a 47,1\%, posicionándonos como el segundo país de la Organización para la Cooperación y el Desarrollo Económicos (OCDE) con mayor número de partos por cesáreas, con diferencias marcadas dependiendo del sistema de salud analizado, con tasas de $40,5 \%$ en los hospitales públicos y $70 \%$ aproximadamente en las clínicas privadas del país.(3) Es muy probable que cualquier intervención que disminuya los costos de la cesárea, tendrá un gran impacto en la salud pública.

En 1990, el Dr. Henrik Kehlet propuso una metodología que permitiera disminuir el estrés de la cirugía de colon por cáncer, buscando modificar las respuestas fisiológicas y psicológicas post operatorias para poder tener un post operatorio más rápido, sin afectar la morbi-mortalidad,(5) lo que hoy se conoce como Enhanced Recovery After Surgery (ERAS). En diciembre de 2018 se publicaron las recomendaciones de la Sociedad ERAS para aplicar ERAS en cesárea(6)(7)(8), tratando de homologar la experiencia seminal de Kehlet.

Nuestro objetivo es realizar un análisis crítico de las recomendaciones propuestas y ver la aplicabilidad de estas guías en Chile. Además, revisamos la experiencia de países que ya han comenzado con la implementación de ERAS en cesárea y de la percepción de diferentes actores sobre la factibilidad de su aplicación en nuestra realidad.

\section{Revisión}

Se analizó críticamente las guías de la Sociedad de Recuperación Temprana post Quirúrgica (ERAS Society) sobre el preoperatorio,(6) intraoperatorio (7) y postoperatorio (8) de ERAS para cesárea. Asimismo, se examinó la bibliografía sobre la cual los autores se basaron para emitir las recomendaciones.

La Sociedad ERAS confecciona sus guías seleccionando un grupo de expertos que realiza una revisión de la literatura y selecciona artículos relevantes. Posteriormente, califican la calidad de evidencia y las recomendaciones según la metodología GRADE.(9) La evidencia se califica como de "alta", "moderada", "baja" o "muy baja" calidad dependiendo si la aparición de nueva evidencia cambiaría las conclusiones de la literatura actual. A su vez, las recomendaciones de la guía se califican como "fuerte" o "débil", dependiendo de la calidad de la evidencia y de los beneficios de la intervención. 
A continuación, describiremos los aspectos fundamentales que considera ERAS para cesárea en los períodos pre, intra y post operatorios.

\section{Preoperatorio}

Premedicación: Se recomienda fuertemente el uso de antiácidos y antagonistas del receptor $\mathrm{H} 2$ de histamina en todas las pacientes para disminuir el riesgo de neumonitis por aspiración de contenido gástrico. También recomiendan fuertemente evitar la sedación perioperatoria en cesárea electiva.

La recomendación del uso de antiácidos se fundamenta en una revisión Cochrane (10) que solo involucraba reportes de mujeres sometidas a cesárea bajo anestesia general. El estudio tiene una baja calidad de evidencia y los hallazgos indican que la combinación de antiácidos más antagonistas $\mathrm{H} 2$ fue más eficaz que ninguna intervención y superior a los antiácidos solos para prevenir un bajo $\mathrm{pH}$ gástrico. Ninguno de los estudios evaluó efectos adversos potenciales o desenlaces clínicos relevantes.(10)

Preparación colónica: La guía recomienda fuertemente no realizar preparación colónica para la operación cesárea. La preparación intestinal preoperatoria oral y/o mecánica se ha utilizado principalmente en cirugía colorrectal para prevenir infecciones posoperatorias y filtración de las anastomosis. Sin embargo, un reciente metaanálisis que incluyó estudios de cirugía ginecológica no encontró beneficio a la preparación intestinal. El único efecto claro fue provocar una "experiencia más desagradable para la paciente". Solo hay un pequeño ensayo clínico de preparación intestinal mecánica antes de la cesárea, que no documentó beneficio(11).

Ayuno: La guía recomienda fuertemente cumplir ayuno de 6 horas para un régimen liviano y 2 horas para líquidos claros. Esta recomendación se apoya en las guías de ayuno de las Sociedades Americana (ASA) y Europea (ESA) de Anestesiología.(12)

Suplementación preoperatoria de carbohidratos: La guía recomienda débilmente la suplementación con carbohidratos por vía oral 2 horas previo a la cesárea, en pacientes no diabéticas.

Una revisión Cochrane (13) demostró que el tratamiento preoperatorio con carbohidratos se asoció con una reducción pequeña en la duración de la estancia hospitalaria en comparación con placebo o ayuno, en pacientes adultos a los que se les realizó cirugía electiva (0,30 días; intervalo de confianza 95\% [IC95\%]: 0,04 a 0,56 días; evidencia de calidad muy baja). Se encontró que el tratamiento preoperatorio con carbohidratos no influyó en las tasas de complicaciones post operatorias en comparación con placebo o ayuno. Esta revisión no incluyó pacientes sometidas a operación cesárea. Por el momento no existe un estudio que demuestre beneficio de suplementar carbohidratos orales en mujeres embarazadas sometidas a cesárea. Esta recomendación, si es que se pone en práctica, debe atenerse a las guías de ayuno actuales.

\section{Intraoperatorio}

Profilaxis antibiótica: La guía recomienda fuertemente el uso de antibióticos en un período no mayor a 60 minutos previos a la incisión de la piel. La recomendación es administrar una cefalosporina de primera generación. También recomienda fuertemente administrar azitromicina en las pacientes que estaban en trabajo de parto con las membranas rotas.

La recomendación del uso de cefalosporinas se basa en una revisión Cochrane (14) que demostró que las cefalosporinas de primera generación son eficaces para disminuir las infecciones post parto, sin agregar efectos adversos maternos.

La recomendación del uso de azitromicina se basa en un estudio que demostró que la adición de azitromicina a la cefalosporina disminuía las infecciones puerperales de $12 \%$ a $6,1 \%$. (riesgo relativo (RR), 0,51; IC95\%, 0,38 a 0,68; $\mathrm{P}<0,001))$. (15)

Preparación de la piel: Se recomienda que las pacientes se duchen con un jabón antimicrobiano idealmente antes de la admisión al hospital por una cesárea programada. La guía recomienda fuertemente el uso de clorhexidina alcoholada por sobre povidona yodada para la asepsia de la piel previo a la incisión quirúrgica.

La guía recomienda débilmente la preparación vaginal con povidona yodada para disminuir las infecciones postparto. Esta recomendación viene de una revisión Cochrane (16) que concluyó que la preparación vaginal con solución antiséptica inmediatamente antes de la cesárea probablemente reduce la incidencia de endometritis post cesárea de 8,7\% a 3,8\% (RR: 0,36; IC 95\%: 0,20 a 0,63), evidencia de calidad moderada. 
Manejo anestésico: La guía recomienda fuertemente el uso de anestesia regional sobre anestesia general, aunque la evidencia que respalda tal preferencia es baja. Los argumentos a su favor son que tiene un impacto positivo para mejorar los resultados de la recuperación en términos de control del dolor, función de los órganos, movilidad, náuseas y vómitos posoperatorios, estadía intrahospitalaria y eventos adversos.(17) Sin embargo, un meta análisis reportó que no hubo evidencia que la anestesia regional sea superior a la anestesia general en términos de mejores desenlaces maternos o neonatales.(18)

Prevención de hipotermia intraoperatoria: La guía recomienda fuertemente monitorizar la temperatura corporal, además de usar tres métodos de prevención de hipotermia conjuntamente como: calentador de fluidos, frazada de aire forzado y temperatura de pabellón igual o mayor a $23^{\circ} \mathrm{C}$. La recomendación se basa en estudios que demuestran que la hipotermia central se asocia a complicaciones en pacientes no obstétricas, tales como: calofríos, infección de sitio operatorio, coagulopatía, sangrado y recuperación prolongada.(19)(20) Un meta análisis realizado por Sultan y cols. concluyó que la mantención activa de la temperatura corporal disminuyó la hipotermia (RR 0,66, IC95\% [0,50, 0,87]; $\mathrm{P}=0,003$ ), los calofríos (RR 0,58 IC95\% [0,43, 0,79]; $\mathrm{P}=0,0004$ ) y logró un mayor $\mathrm{pH}$ umbilical (diferencia media $0,02, \mathrm{IC} 95 \%[0,0,05] ; \mathrm{P}=0,04)$ en cesáreas electivas.(21) La recomendación del uso de tres métodos diferentes para prevenir la hipotermia se basa en estudios que demostraron que el uso de dos métodos diferentes tenían un impacto modesto en la mantención de la normotermia.(22)

Técnicas quirúrgicas de la cesárea: La guía entrega cinco consideraciones para la técnica quirúrgica de la cesárea, cuyos grados de recomendación es débil. Las recomendaciones tienen el objetivo de disminuir las complicaciones, más que para disminuir el tiempo de estadía hospitalaria.

\section{Las recomendaciones son:}

1 Expansión manual de la histerotomía hacia lateral (para disminuir sangrado).

2 Histerorrafia en dos capas (para disminuir riesgo de rotura uterina).

3 No cerrar peritoneo.

4 Aproximar tejido subcutáneo en pacientes con subcutáneo sobre $2 \mathrm{~cm}$.

5 Cierre de piel con sutura intradérmica.

Manejo de fluidos en el perioperatorio: La guía recomienda fuertemente la mantención de la euvolemia en el perioperatorio. Esta recomendación viene de un consenso internacional para el manejo de hipotensión arterial en cesárea, que recalca la limitada eficacia de los fluidos intravenosos por sí solos para evitar la hipotensión arterial y recomienda el uso de drogas vasoactivas con efecto alfa agonista, asociadas a cohidratación y desplazamiento uterino.(23) La euvolemia preoperatoria e intraoperatoria son factores importantes en la atención perioperatoria de la paciente y parecen conducir a mejores desenlaces maternos y neonatales después de la cesárea.

Atención neonatal: La guía entrega seis recomendaciones fuertes sobre la atención neonatal.

1 Pinzamiento tardío de al menos un minuto para recién nacidos de término.

2 Pinzamiento tardío de al menos 30 segundos para recién nacidos de pretérmino.

3 Mantener la temperatura corporal entre $36,5^{\circ} \mathrm{C}$ y $37,5^{\circ} \mathrm{C}$.

4 Evitar succión de la vía aérea o aspiración gástrica si no es necesario.

5 Suplementar con aire ambiental y no oxígeno si es posible.

6 Tener la capacidad de hacer una resucitación neonatal si fuese necesario.

\section{Postoperatorio}

Alimentación placebo (uso de goma de mascar): La guía recomienda débilmente fomentar el masticar goma de mascar, especialmente en pacientes donde el ayuno post operatorio será más prolongado. Esta recomendación se basa en cuatro trabajos que demostraron que mascar chicle disminuye el íleo post operatorio, pero no disminuye el tiempo de hospitalización. 
Manejo de náuseas y vómitos post operatorios: La guía recomienda fuertemente prevenir la hipotensión arterial intraoperatoria, así como la prevención de náuseas y vómitos post operatorios con un enfoque multimodal.

Analgesia: La guía recomienda fuertemente un enfoque multimodal de analgesia centrado en antiinflamatorios no esteroidales (AINES) y paracetamol, como complemento al uso de morfina neuroaxial o bloqueos de pared como, por ejemplo, el bloqueo del plano del transverso abdominal (TAP). Se hace la advertencia que el uso de morfina intratecal puede aumentar el riesgo de aparición de efectos adversos como náuseas, vómitos y prurito.

En Chile, habitualmente se utiliza morfina intratecal, así como AINES y paracetamol para el manejo de dolor post operatorio. Recientemente fue publicado por la Sociedad Americana de Anestesia Obstétrica y Perinatológica (SOAP) las recomendaciones sobre el uso de morfina neuroaxial para analgesia post cesárea.(24) En ellas por primera vez se ha recomendado que, en pacientes sin factores de riesgo, el uso de morfina en bajas dosis (menos de $50 \mathrm{ug}$ intratecal o menos de $1 \mathrm{mg}$ epidural), no se requiere de control de frecuencia respiratoria horaria, mientras que con dosis entre 50 a 150 ug intratecal y 1 a $3 \mathrm{mg}$ epidural, el control de frecuencia respiratoria es cada 3 horas por 12 horas. Esta nueva recomendación probablemente hará cambiar la conducta en muchos lugares hacia un mayor uso de morfina neuroaxial.

Cuidados nutricionales perioperatorios/Alimentación temprana: La guía recomienda fuertemente que se inicie alimentación regular a las dos horas del post operatorio. Múltiples estudios han demostrado que la realimentación precoz (a las 2 horas de la cirugía) provee similar o mayor satisfacción materna, reanudación más temprana del consumo de alimentos sólidos, reinicio más rápido de la actividad intestinal y reducción de la estadía hospitalaria, sin aumentar las tasas de complicaciones relacionadas con la herida.

Prevención de tromboembolismo: La guía recomienda fuertemente el uso de medias anti embólicas en todas las pacientes que vayan a cesárea. Por otro lado, recomienda débilmente la no utilización de trombo profilaxis farmacológica. Esta recomendación proviene de una revisión Cochrane(25) que no fue concluyente sobre la utilidad del uso de la tromboprofilaxis en cesárea.

Movilización temprana: La guía recomienda débilmente la movilización temprana en el post operatorio de la cesárea. La recomendación débil se debe a la poca evidencia que se cuenta sobre los beneficios de la movilización temprana.

Manejo del drenaje urinario: La guía recomienda fuertemente el retiro de la sonda vesical en el post operatorio inmediato. Esta recomendación se justifica dado que las infecciones del tracto urinario son una de las complicaciones más comunes después de la cesárea. Una revisión Cochrane (26) encontró que el cateterismo vesical no disminuye las lesiones intraoperatorias de la vejiga, como tampoco reduce la retención urinaria en el post operatorio. La publicación también concluye que los estudios existentes son de baja calidad.

\section{Experiencia Internacional}

En países donde la salud es completamente dependiente del estado, como en el Reino Unido, la necesidad de disminuir los costos ha aumentado el interés de los servicios de salud para reducir los días de hospitalización de la cesárea.(27) A continuación se describen brevemente los resultados de aquella iniciativa.

La implementación de ERAS en cesárea incluyó a todas las pacientes de cesáreas electivas, a las que se les entregó información completa del proceso de ERAS en el preoperatorio. Se les permitió ingerir líquidos claros hasta dos horas previo a la cirugía y se les incentivó a ingerir una bebida deportiva isotónica dos horas previo a la cirugía. En el intraoperatorio se manejó la volemia a criterio del anestesista, la técnica quirúrgica fue a discreción del cirujano y se mantuvo la temperatura con aire forzado con manta de hemicuerpo inferior. En el postoperatorio, la paciente se movilizó y se retiró la sonda vesical al día siguiente de la cirugía. La analgesia se logró con opioides intratecales, paracetamol y AINES. La prevención de náuseas y vómitos fue a discreción del equipo y se decidió realimentar 1 hora posterior a la cirugía.

$\mathrm{Al}$ inicio del estudio, solo 1,6\% de las pacientes egresaban del hospital en el primer día postoperatorio. Al fin del estudio $25,2 \%$ de las pacientes lograron egresar el primer día. Interesantemente, el mayor aumento de egresos en el primer día sucedió luego de comenzar con el aumento de información a la paciente sobre el protocolo ERAS.

Luego de la implementación del protocolo ERAS en cesárea, los autores no observaron mayor readmisión al hospital de pacientes que egresaron al hospital en el primer día postoperatorio versus las pacientes que egresaron en el segundo día. Una encuesta demostró que la analgesia y capacidad de realizar actividades fue igual en ambos grupos. 
Los autores refieren que diferentes situaciones neonatales fueron determinantes en la estancia materna en el hospital.

\section{Experiencia Nacional}

Para conocer la experiencia nacional actual respecto a ERAS en cesárea, realizamos una encuesta online a través de la Sociedad de Anestesia de Chile (SACH), la Sociedad Chilena de Obstetricia y Ginecología (SOCHOG) y los neonatólogos asociados a la Sociedad Chilena de Pediatría (SOCHIPE).

La encuesta fue respondida por 153 médicos, donde $66 \%$ fueron anestesiólogos, $18 \%$ obstetras y $6 \%$ otros médicos. La distribución por sexo de los participantes fue de 93 hombres (60\%) y 57 mujeres (40\%).

$28 \%$ de los encuestados ya realiza ERAS en operación cesárea. En estos centros se cumplen al menos $50 \%$ de las recomendaciones del protocolo de la Sociedad ERAS, mientras que en los centros donde no se realiza ERAS, se cumplen en menor cantidad dichas recomendaciones.

En aquellos centros en que se hace ERAS para cesárea, los aspectos en que la técnica hace hincapié y que se cumplen en más de 50\% de los casos son 8 de 18 aspectos: evaluación preoperatoria (77\%), uso de antibióticos (83\%), anestesia espinal con opioides (64\%), pinzamiento del cordón tardío (60\%), apego y lactancia intraoperatoria (57\%), analgesia oral y multimodal (65\%), movilización precoz (75\%) y retiro precoz de la sonda vesical $(65 \%)$. A su vez, en aquellos que no se hace ERAS, sólo se hacen 4 de 18 aspectos: uso de antibióticos (85\%), anestesia espinal con opioides (74\%), analgesia oral y multimodal (66\%), movilización precoz (60\%). Hay una percepción de que se consideran más aspectos de lo que se esperaría hacer teniendo una lista de verificación. De hecho, aquellos centros que hacen ERAS tienen más auditorías de los casos que aquellos que no (15 de 36 (42\%) versus 4 de 84 (5\%), p<0,001, test de Fisher).

Los aspectos en que hay más control cuando se realiza ERAS son: retiro de la sonda vesical precoz (a las 7 horas) (28 de $36(78 \%)$ versus 32 de $84(38 \%), p=0,032$, test de Fisher) y mayor asesoría en la lactancia materna (16 de 36 (44\%) versus 20 de 84 (24\%), p=0,025, test de Fisher). En los otros aspectos no hay diferencias al realizar un protocolo ERAS versus uno tradicional en la muestra obtenida en la encuesta.

En opinión de los encuestados, las opciones que consideran como un aporte de la técnica ERAS en cesárea son: beneficio materno (satisfacción): 34 de 140 (24\%); beneficio neonatal (mayor probabilidad de apego materno y/o lactancia materna efectiva): 33 de 140 (24\%); menores complicaciones: 35 de 140 (25\%) y menor costo total: 20 de 140 $(14 \%)$.

Los encuestados opinaron que los cinco factores limitantes más importantes para incorporar la técnica son: miedo a lo nuevo: 71 de 144 (49\%); deseos de las pacientes de quedarse más días hospitalizada: 48 de 144 (33\%); deseos del obstetra/necesidad de la paciente: 46 de 144 (32\%); cobertura por pagador de salud de hospitalización habitual: 33 de 144 (23\%) y; más trabajo del equipo de salud: 30 de 144 (21\%).

Finalmente, sobre la pregunta si los médicos recomendarían el protocolo ERAS en cesárea para ellos mismos o a un familiar, $67 \%$ respondió positivamente. Los médicos que sí realizan ERAS en su centro recomendarían más frecuentemente el protocolo versus lo que no la realizan (34 de 36 (94\%) vs 51 de 87 (58\%), p <0,001, test de Fisher).

Otro ejemplo de experiencia internacional proviene de California, EE.UU. Donde la Dra. Lee(28) implementó un modelo de protocolo ERAS en cesárea en 1390 cesáreas electivas y de urgencia. Se demostró que la implementación del protocolo ERAS aumentó la deambulación de 33\% a 51\% ( $<<0.001)$, aumento la realimentación precoz de $17 \%$ a $57 \%$ ( $\mathrm{p}<0.001)$ y disminuyó el consumo postoperatorio de opioides de $13.1 \mathrm{mg}$ de morfina a $7.7 \mathrm{mg}(\mathrm{p}<0.001)$.

\section{Discusión}

Los protocolos ERAS han llegado para quedarse. En un futuro cercano, es posible que los hospitales, las autoridades en salud, las aseguradoras o los mismos pacientes exijan los protocolos. Actualmente, se están formando equipos de ERAS en cada continente y se han comenzado a realizar congresos mundiales. Debido a la alta carga asistencial en un sistema con bajos recursos económicos, Chile y Latinoamérica podrían ser beneficiados de protocolizar la atención en salud.

El protocolo ERAS tiene como objetivo estandarizar la atención perioperatoria, reduciendo la variabilidad en la atención, favoreciendo la creación de una vía de atención específica basada en la evidencia para el progreso de los resultados maternos y fetales, apuntando a la mejoría continua del proceso. 
El éxito de la implementación de ERAS puede no necesariamente llevar a una reducción en la duración de la estadía de la madre y/o recién nacido o ser una intervención de reducción de costos, más bien la misión de ERAS es mejorar la calidad global de la atención y optimizar la calidad de la recuperación después del parto por cesárea(24).

Al revisar los protocolos ERAS, la impresión inicial es que muchas de las acciones recomendadas ya están instauradas en los propios centros y que esta nueva forma de trabajo no aportaría mayormente para lograr una mejor experiencia para las pacientes, médicos o los sistemas de salud. Si bien, algo de esta percepción es cierta, el aporte principal del protocolo ERAS es a ordenar y estandarizar un proceso complejo, basado en la mejor evidencia disponible y realizar un seguimiento de los resultados que permita hacer modificaciones y mejoras continuas, sin quedarse en la idea que el único objetivo es la reducción en los días de estadía hospitalaria.

La iniciativa ERAS para cesárea tiene algunas limitaciones. Así como los encuestados lo perciben, las principales son: miedo a lo nuevo, deseos personales y conflictos con los pagadores de salud. Sobre la primera, es muy posible que el sólo hecho de educar e informar a los principales grupos de interés hará que esta limitante disminuya en forma considerable. Sobre los deseos personales, tanto de pacientes como de médicos, una vez que se transparenten resultados clínicos y económicos, puede que esta limitante retroceda. Por otro lado, las expectativas de la madre, como las del ginecólogo-obstetra podrían ser una gran limitante en la implementación del protocolo, si es que no se realiza una educación e información adecuada.

Como se observa en la experiencia internacional, el neonato es un factor importante en el retraso del egreso hospitalario de la madre. En Chile, muchas madres sienten un alivio al estar hospitalizadas los primeros días para así tener un apoyo inicial con el recién nacido. Sería importante organizar una red de soporte, telefónico o de visita, para apoyar a las madres o pesquisar neonatos con alguna patología de reciente comienzo.

Finalmente, es importante conocer la disposición de los prestadores públicos y privados del país, dado que una disminución en la estadía hospitalaria podría disminuir el pago de las aseguradoras en salud. En Chile, habitualmente los prestadores de salud proponen un valor fijo a las aseguradoras. Si es que no hay beneficio para los prestadores, el protocolo difícilmente será implementado.

En resumen, creemos que esta forma de trabajo es muy interesante y que puede ser un aporte real en la atención de las embarazadas que requieren cesárea. La opinión mayoritaria de los encuestados fue que recomendarían el protocolo ERAS a un ser querido, lo que reafirma nuestra percepción a que es un real aporte. Para nosotros, el protocolo ERAS en cesárea no implica grandes diferencias con el manejo habitual, por lo que el rol del anestesiólogo no debería cambiar mayormente.

En Chile, es importante comenzar a educar e informar a la población precozmente si es que se quiere comenzar con ERAS en cesárea en el mediano plazo y posiblemente un protocolo de ERAS para parto en el largo plazo. Esta educación debe ser acompañada con un fuerte énfasis en el apoyo a la madre en el ambulatorio, especialmente enfocada en la salud del neonato. Para poder comenzar a implementar el protocolo ERAS en Chile se debe incorporar a la salud primaria, con una buena captación y educación de paciente en el ante operatorio y una buena red de apoyo y atención en el postoperatorio que se realice en domicilio.

\section{$\underline{\text { Referencias }}$}

1. C AB. Relationship between cesarean delivery rate and maternal and neonatal mortality: Editorial comment. Obstet Gynecol Surv. 2016;71(4):201-2.

2. Souza J, Gulmezoglu AM, Lumbiganon P, Laopaiboon M, Carrin G, Fawole B, et al. Who Global Survey on Maternal and Perinatal Health. BMC Med. 2010;8(71):1-10.

3. Magne F, Silva AP, Carvajal B, Gotteland M. The elevated rate of cesarean section and its contribution to non-communicable chronic diseases in Latin America: The growing involvement of the microbiota. Front Pediatr. 2017;5(September).

4. Borrescio-Higa F, Valdés N. Publicly insured caesarean sections in private hospitals: A repeated cross-sectional analysis in Chile. BMJ Open. 2019;9(4):1-7.

5. Kehlet H. Multimodal approach to control postoperative pathophysiology and rehabilitation. Br J Anaesth. 1997;78(5):606-17. 
6. Wilson RD, Caughey AB, Wood SL, Macones GA, Wrench IJ, Huang J, et al. Guidelines for Antenatal and Preoperative care in Cesarean Delivery: Enhanced Recovery After Surgery Society Recommendations (Part 1). Am J Obstet Gynecol [Internet]. 2018;219(6):523.e1-523.e15. Available from: https://doi.org/10.1016/j.ajog.2018.09.015

7. Caughey AB, Wood SL, Macones GA, Wrench IJ, Huang J, Norman M, et al. Guidelines for intraoperative care in cesarean delivery: Enhanced Recovery After Surgery Society Recommendations (Part 2). Am J Obstet Gynecol [Internet]. 2018;219(6):533-44. Available from: https://doi.org/10.1016/j.ajog.2018.08.006

8. Macones GA, Caughey AB, Wood SL, Wrench IJ, Huang J, Norman M, et al. Guidelines for postoperative care in cesarean delivery: Enhanced Recovery After Surgery (ERAS) Society recommendations (part 3). Am J Obstet Gynecol [Internet]. 2019;221(3):247.e1-247.e9. Available from: https://doi.org/10.1016/j.ajog.2019.04.012

9. Guyatt GH, Oxman AD, Vist GE, Kunz R, Falck-Ytter Y, Alonso-Coello P, et al. GRADE: An emerging consensus on rating quality of evidence and strength of recommendations. Chinese J Evidence-Based Med. 2009;9(1):8-11.

10. Paranjothy S, Griffiths JD, Broughton HK, Gyte GML, Brown HC, Thomas J. Interventions at caesarean section for reducing the risk of aspiration pneumonitis. Cochrane Database Syst Rev. 2014;2014(2).

11. Lurie S, Baider C, Glickman H, Golan A, Sadan O. Are enemas given before cesarean section useful? A prospective randomized controlled study. Eur J Obstet Gynecol Reprod Biol [Internet]. 2012;163(1):27-9. Available from: http://dx.doi.org/10.1016/j.ejogrb.2012.03.034

12. Smith I, Kranke P, Murat I, Smith A, O’Sullivan G, Søreide E, et al. Perioperative fasting in adults and children: Guidelines from the european society of anaesthesiology. Eur J Anaesthesiol. 2011;28(8):556-69.

13. Mccall J, Plank L, Gp H, Soop M, Nygren J. Preoperative carbohydrate treatment for enhancing recovery after elective surgery ( Review ) SUMMARY OF FINDINGS FOR THE MAIN COMPARISON. Cochrane Collab Cochrane Database Syst Rev. 2014;(8).

14. Mackeen AD, Packard RE, Ota E, Berghella V, Baxter JK. Timing of intravenous prophylactic antibiotics for preventing postpartum infectious morbidity in women undergoing cesarean delivery. Cochrane Database Syst Rev. 2014;2014(12).

15. Tita ATN, Szychowski JM, Boggess K, Saade G, Longo S, Clark E, et al. Adjunctive azithromycin prophylaxis for cesarean delivery. N Engl J Med. 2016;375(13):1231-41.

16. Haas DS, Morgan S, Contreras K, Enders S. for preventing postoperative infections ( Review ). Cochrane Database Syst Rev. 2018;7.

17. McIsaac DI, Cole ET, McCartney CJL. Impact of including regional anaesthesia in enhanced recovery protocols: A scoping review. Br J Anaesth [Internet]. 2015;115:ii46-56. Available from: http://dx.doi.org/10.1093/bja/aev376

18. Afolabi BB LF. Regional versus general anaesthesia for caesarean section. Cochrane Database Syst Rev. 2012;10:CD00435.

19. Kurz A, Sessler DI, Lenhardt R. Perioperative normothermia to reduce the incidence of surgical-wound infection and shorten hospitalization. N Engl J Med. 1996;334(19):1209-15.

20. Tommasino C. Mild hypothermia. J Neurosurg Sci. 1998;42(1 SUPPL. 1):37-8.

21. Sultan P, Habib AS, Cho Y, Carvalho B. The Effect of patient warming during Caesarean delivery on maternal and neonatal outcomes: A meta-analysis. Br J Anaesth. 2015;115(4):500-10.

22. Cobb, B., Cho, Y., Hilton, G., Ting, V., \& Carvalho B. Active Warming Utilizing Combined IV Fluid and ForcedAir Warming Decreases Hypothermia and Improves Maternal Comfort During Cesarean Delivery: A Randomized Control Trial. Anesth Analg. 122(5):1490-1497.

23. Kinsella SM, Carvalho B, Dyer RA, Fernando R, McDonnell N, Mercier FJ, et al. International consensus statement on the management of hypotension with vasopressors during caesarean section under spinal anaesthesia. Anaesthesia. 2018;73(1):71-92.

24. Bauchat JR, Weiniger CF, Sultan P, Habib AS, Ando K, Kowalczyk JJ, et al. Society for Obstetric Anesthesia and Perinatology Consensus Statement: Monitoring Recommendations for Prevention and Detection of Respiratory 
Depression Associated With Administration of Neuraxial Morphine for Cesarean Delivery Analgesia. Anesth Analg. 2019;129(2):458-74.

25. Tooher R, Gates S, Dowswell T, Davis L. Prophylaxis for venous thromboembolic disease in pregnancy and the early postnatal period. In: Cochrane Database of Systematic Reviews. 2010.

26. Abdel-Aleem H, Aboelnasr MF, Jayousi TM, Habib FA. Indwelling bladder catheterization as part of postoperative care for caesarean section. Cochrane Database Syst Rev. 2013;2013(1).

27. Wrench IJ, Allison A, Galimberti A, Radley S, Wilson MJ. Introduction of enhanced recovery for elective caesarean section enabling next day discharge: A tertiary centre experience. Int J Obstet Anesth [Internet]. 2015;24(2):124-30. Available from: http://dx.doi.org/10.1016/j.ijoa.2015.01.003

28. Lee KL, Lee DC, Huang M, Hunt EJ, Hedderson MM. Enhanced Recovery after Surgery Implementation in a Cesarean Section Population in an Integrated Healthcare System [35B]. Obstet Gynecol. 2018;131(5):29S. 
